\title{
Kinetic and Donor Stabilization of Organotellurenyl Iodides and Azides
}

Thomas M. Klapötke, * Burkhard Krumm, Heinrich Nöth,

Juan Carlos Gálvez-Ruiz, Kurt Polborn, Ingo Schwab, and Max Suter

\section{Supporting Information}
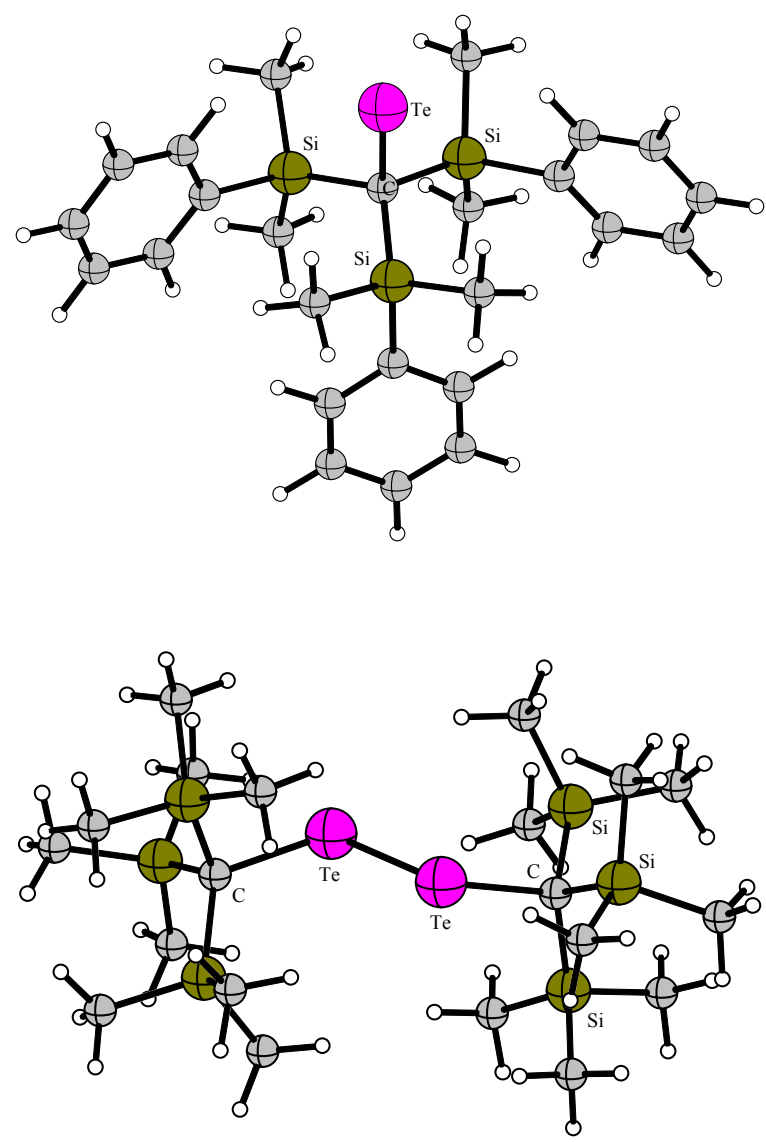

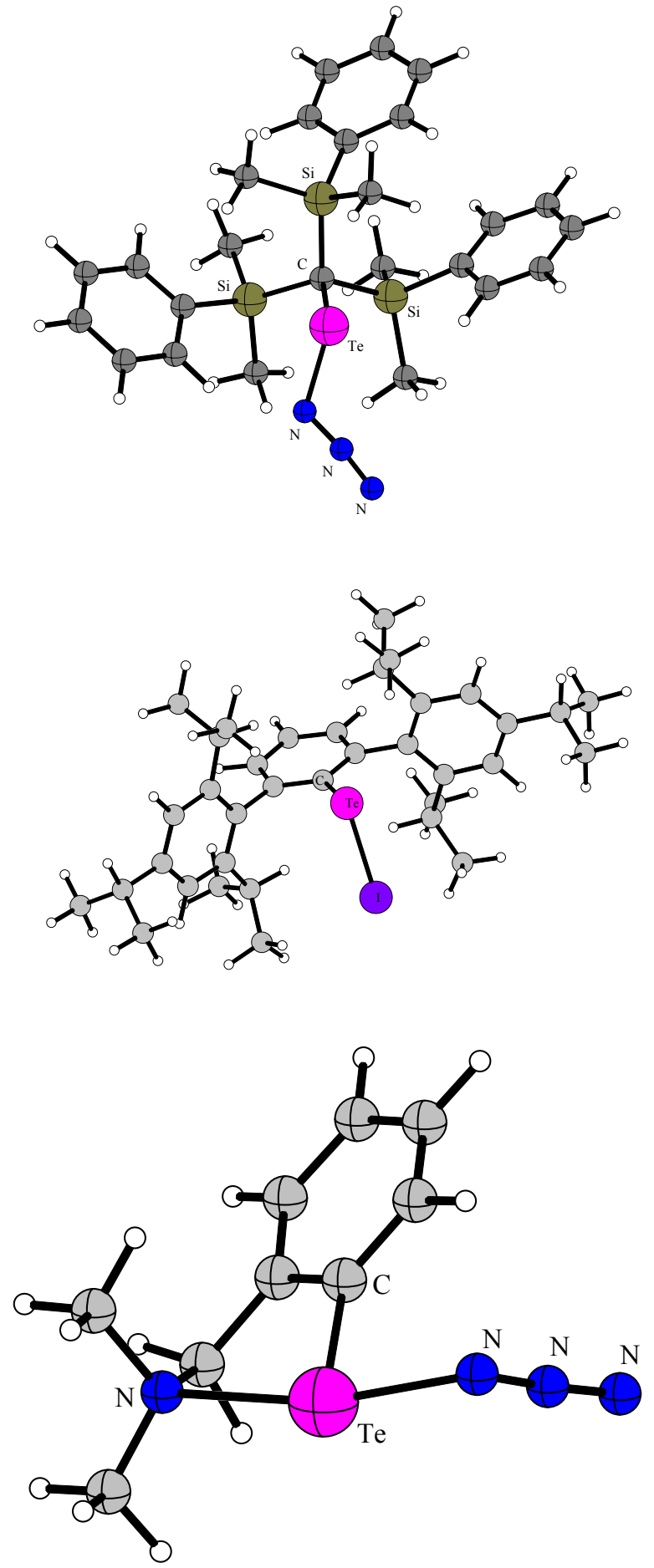

Figure 1. Calculated structures of TpsiTeLi (1), (TsiTe) $)_{2}, \operatorname{TpsiTeN}_{3}(\mathbf{4})$, and 2,6-Trip ${ }_{2} \mathrm{C}_{6} \mathrm{H}_{3} \mathrm{TeI}(\mathbf{1 0})$ at the MARI-J-BP86/TZVP level and 2- $\mathrm{Me}_{2} \mathrm{NCH}_{2} \mathrm{C}_{6} \mathrm{H}_{4} \mathrm{TeN}_{3}$ (12) at the RI-MP2(FC)/TZVPP level of theory. 
Table 1. (TsiTe $)_{2}$ Geometry Optimization

\begin{tabular}{|c|c|c|c|}
\hline E [a.u.] & Job & torsion angle $\mathrm{C}-\mathrm{Te}-\mathrm{Te}-\mathrm{C}\left[^{\circ}\right]$ & $\mathrm{d} \mathrm{Te}-\mathrm{C}[\AA]$ \\
\hline-2548.5684881560 & tsi-170 & 161.81 & Te-C $2.246 / 2.248$ \\
\hline-2548.5684859710 & tsi-160 & 161.40 & $\mathrm{Te}-\mathrm{C}=2.246 / 2.248$ \\
\hline-2548.5684873230 & tsi (screwer 50) & 158.80 & $\mathrm{Te}-\mathrm{C} 2.247 / 2.249$ \\
\hline-2548.5683772240 & tsi (screwer 100) & 166.38 & $\mathrm{Te}-\mathrm{C} 2.245 / 2.246$ \\
\hline-2548.5684898080 & tsi (screwer 150) & 158.74 & Te-C 2.247 / 2.249 \\
\hline-2548.5685018330 & tsi (50tight) & 160.14 & $\mathrm{Te}-\mathrm{C} 2.247 / 2.248$ \\
\hline-2548.5683834620 & tsi (100tight) & 165.82 & $\mathrm{Te}-\mathrm{C} 2.245 / 2.246$ \\
\hline-2548.5684917830 & tsi (150tight) & 158.83 & $\mathrm{Te}-\mathrm{C} 2.247 / 2.249$ \\
\hline-2548.5684813380 & tsi-cosmo & 179.60 & $1 \mathrm{c}-2 \mathrm{te}=2.246$ \\
\hline
\end{tabular}

Cartesian coordinates of the minimum structures for TpsiTeLi (1), $\operatorname{TpsiTeN}_{3}$ (4), 2,6$\operatorname{Trip}_{2} \mathrm{C}_{6} \mathrm{H}_{3} \mathrm{TeI}(\mathbf{1 0})$ and 2- $\mathrm{Me}_{2} \mathrm{NCH}_{2} \mathrm{C}_{6} \mathrm{H}_{4} \mathrm{TeN}_{3}$ (12):

\begin{tabular}{|c|c|c|c|}
\hline \multicolumn{4}{|l|}{62} \\
\hline Tps & e- anion, & RI - BP $86 / T Z V P$, & Energy $=-1849.742257570$ \\
\hline $\mathrm{Te}$ & -2.3341820 & -0.1263566 & 0.5801003 \\
\hline $\mathrm{C}$ & -0.2562286 & -0.0119302 & -0.3751566 \\
\hline $\mathrm{Si}$ & -0.3183348 & 1.5878244 & -1.4431437 \\
\hline $\mathrm{Si}$ & -0.0960595 & -1.6118240 & -1.4322689 \\
\hline $\mathrm{Si}$ & 0.9075968 & 0.0520557 & 1.1613565 \\
\hline $\mathrm{C}$ & 0.7705802 & -1.4721413 & 2.2960965 \\
\hline $\mathrm{H}$ & 1.4029192 & -1.3003069 & 3.1818497 \\
\hline $\mathrm{H}$ & -0.2745330 & -1.5779510 & 2.6174347 \\
\hline $\mathrm{H}$ & 1.0758392 & -2.4155971 & 1.8274054 \\
\hline C & 0.6274038 & 1.5475830 & 2.3082166 \\
\hline $\mathrm{H}$ & -0.4274438 & 1.5714090 & 2.6140420 \\
\hline $\mathrm{H}$ & 1.2570001 & 1.4111368 & 3.2020048 \\
\hline $\mathrm{H}$ & 0.8741444 & 2.5151836 & 1.8549141 \\
\hline C & 1.5550859 & -1.7094331 & -2.4096926 \\
\hline $\mathrm{H}$ & 2.4439865 & -1.5726633 & -1.7785439 \\
\hline $\mathrm{H}$ & 1.6296235 & -2.6880579 & -2.9098693 \\
\hline $\mathrm{H}$ & 1.5886539 & -0.9378775 & -3.1902068 \\
\hline C & -1.4586674 & -1.7852236 & -2.7613548 \\
\hline $\mathrm{H}$ & -2.4392543 & -1.5117977 & -2.3480267 \\
\hline $\mathrm{H}$ & -1.2533793 & -1.1536894 & -3.6380440 \\
\hline $\mathrm{H}$ & -1.4941442 & -2.8326361 & -3.0979745 \\
\hline C & 1.2138370 & 1.7884141 & -2.5879253 \\
\hline $\mathrm{H}$ & 1.2248252 & 2.8009664 & -3.0210479 \\
\hline $\mathrm{H}$ & 2.1714560 & 1.6162160 & -2.0772975 \\
\hline $\mathrm{H}$ & 1.1524735 & 1.0802281 & -3.4264637 \\
\hline C & -1.8047182 & 1.7019463 & -2.6362984 \\
\hline $\mathrm{H}$ & -1.7600946 & 0.9411717 & -3.4271280 \\
\hline
\end{tabular}




$\begin{array}{rrrr}\mathrm{H} & -2.7487431 & 1.5747289 & -2.0906237 \\ \mathrm{H} & -1.7900748 & 2.6959500 & -3.1110830 \\ \mathrm{C} & 2.7883098 & 0.1521559 & 0.6880026 \\ \mathrm{C} & 3.6020478 & -0.9976701 & 0.6355327 \\ \mathrm{C} & 3.4212807 & 1.3867819 & 0.4387261 \\ \mathrm{C} & 4.9677982 & -0.9256250 & 0.3374347 \\ \mathrm{H} & 3.1612826 & -1.9761625 & 0.8419852 \\ \mathrm{C} & 4.7856756 & 1.4722905 & 0.1394021 \\ \mathrm{H} & 2.8368979 & 2.3088471 & 0.4900965 \\ \mathrm{C} & 5.5660775 & 0.3127540 & 0.0837722 \\ \mathrm{H} & 5.5671141 & -1.8393693 & 0.3054933 \\ \mathrm{H} & 5.2417607 & 2.4478767 & -0.0484715 \\ \mathrm{H} & 6.6319686 & 0.3744754 & -0.1483735 \\ \mathrm{C} & -0.3905306 & 3.2320315 & -0.4366786 \\ \mathrm{C} & -1.5480289 & 3.6117223 & 0.2754916 \\ \mathrm{C} & 0.6799283 & 4.1479748 & -0.4586445 \\ \mathrm{C} & -1.6193952 & 4.8364268 & 0.9479403 \\ \mathrm{H} & -2.3856847 & 2.9107875 & 0.3278904 \\ \mathrm{C} & 0.6151079 & 5.3761065 & 0.2114231 \\ \mathrm{H} & 1.5909639 & 3.9027273 & -1.0097472 \\ \mathrm{C} & -0.5384023 & 5.7239105 & 0.9207142 \\ \mathrm{H} & -2.5255669 & 5.0965806 & 1.5010559 \\ \mathrm{H} & 1.4670285 & 6.0604840 & 0.1791240 \\ \mathrm{H} & -0.5944442 & 6.6801167 & 1.4471168 \\ \mathrm{C} & -0.1542218 & -3.2675706 & -0.4433571 \\ \mathrm{C} & 0.9797815 & -4.0998535 & -0.3605079 \\ \mathrm{C} & -1.3463481 & -3.7440345 & 0.1415731 \\ \mathrm{C} & 0.9399222 & -5.3403108 & 0.2884143 \\ \mathrm{H} & 1.9221337 & -3.7765393 & -0.8088166 \\ \mathrm{C} & -1.3927754 & -4.9810997 & 0.7938434 \\ \mathrm{H} & -2.2357037 & -3.1093646 & 0.1132692 \\ \mathrm{C} & -0.2508136 & -5.7851078 & 0.8708037 \\ \mathrm{H} & 1.8404411 & -5.9581191 & 0.3391837 \\ \mathrm{H} & -2.3287560 & -5.3171158 & 1.2475213 \\ \mathrm{H} & -0.2877716 & -6.7508725 & 1.3813359\end{array}$

65 TpsiTeN, MARI-BP86/TZVP, Energy = -2013.928350396

$\begin{array}{lrrr}\mathrm{Te} & -1.4142709 & 1.0465148 & 0.9836499 \\ \mathrm{C} & 0.1355611 & 0.1253604 & -0.3218159 \\ \mathrm{Si} & 1.0573319 & 1.5236055 & -1.3564309 \\ \mathrm{Si} & -0.7692075 & -1.2001016 & -1.4383588 \\ \mathrm{Si} & 1.2776842 & -0.6727133 & 1.0742121 \\ \mathrm{C} & 0.2092589 & -1.3072718 & 2.5278315 \\ \mathrm{H} & 0.8788664 & -1.8330763 & 3.2265685 \\ \mathrm{H} & -0.2505401 & -0.4891854 & 3.1060716 \\ \mathrm{H} & -0.5835891 & -2.0030855 & 2.2246656 \\ \mathrm{C} & 2.4800200 & 0.5872990 & 1.8272711 \\ \mathrm{H} & 1.9662748 & 1.5068557 & 2.1377201 \\ \mathrm{H} & 2.9372999 & 0.1262060 & 2.7162201 \\ \mathrm{H} & 3.2931211 & 0.8721185 & 1.1478046 \\ \mathrm{C} & 0.4168840 & -1.8426246 & -2.7829789 \\ \mathrm{H} & 1.3447099 & -2.2588248 & -2.3669250 \\ \mathrm{H} & -0.0858796 & -2.6329982 & -3.3604655 \\ \mathrm{H} & 0.6859302 & -1.0453718 & -3.4896731 \\ \mathrm{C} & -2.3086475 & -0.5010344 & -2.3107511 \\ \mathrm{H} & -3.1396306 & -0.3474539 & -1.6093554 \\ \mathrm{H} & -2.1270234 & 0.4393889 & -2.8439278 \\ \mathrm{H} & -2.6434593 & -1.2513678 & -3.0436993 \\ \mathrm{C} & 2.6980740 & 0.8741054 & -2.0761986 \\ \mathrm{H} & 3.0382689 & 1.5923751 & -2.8379231\end{array}$




$\begin{array}{rrrr}\mathrm{H} & 3.4964001 & 0.7909811 & -1.3274486 \\ \mathrm{H} & 2.5869434 & -0.1040644 & -2.5594972 \\ \mathrm{C} & 0.0186927 & 2.0921759 & -2.8454430 \\ \mathrm{H} & -0.1533492 & 1.2909327 & -3.5771572 \\ \mathrm{H} & -0.9517477 & 2.4991901 & -2.5333944 \\ \mathrm{H} & 0.5775762 & 2.8944741 & -3.3508860 \\ \mathrm{C} & 2.3195747 & -2.1728854 & 0.4949281 \\ \mathrm{C} & 1.9384160 & -3.4833492 & 0.8451630 \\ \mathrm{C} & 3.5595418 & -2.0150339 & -0.1559586 \\ \mathrm{C} & 2.7472288 & -4.5846239 & 0.5455315 \\ \mathrm{H} & 0.9940860 & -3.6565887 & 1.3620768 \\ \mathrm{C} & 4.3709781 & -3.1109413 & -0.4635906 \\ \mathrm{H} & 3.9138260 & -1.0189748 & -0.4222056 \\ \mathrm{C} & 3.9645296 & -4.4026045 & -0.1156545 \\ \mathrm{H} & 2.4239485 & -5.5871562 & 0.8328629 \\ \mathrm{H} & 5.3259970 & -2.9541855 & -0.9690154 \\ \mathrm{H} & 4.5976758 & -5.2603670 & -0.3501897 \\ \mathrm{~N} & -2.3468128 & 2.4291550 & -0.3434699 \\ \mathrm{~N} & -3.5290651 & 2.2181774 & -0.6236061 \\ \mathrm{~N} & -4.6297695 & 2.1472165 & -0.9492093 \\ \mathrm{C} & 1.4820179 & 3.1271300 & -0.3951957 \\ \mathrm{C} & 0.4901863 & 4.0754969 & -0.0691392 \\ \mathrm{C} & 2.8186995 & 3.4681613 & -0.1097212 \\ \mathrm{C} & 0.8182061 & 5.2926501 & 0.5361132 \\ \mathrm{H} & -0.5580795 & 3.8713475 & -0.2956760 \\ \mathrm{C} & 3.1537330 & 4.6854953 & 0.4919335 \\ \mathrm{H} & 3.6261917 & 2.7792826 & -0.3646905 \\ \mathrm{C} & 2.1512471 & 5.6010171 & 0.8224279 \\ \mathrm{H} & 0.0271652 & 6.0047000 & 0.7802634 \\ \mathrm{H} & 4.2000954 & 4.9189734 & 0.6993340 \\ \mathrm{H} & 2.4080009 & 6.5522401 & 1.2926622 \\ \mathrm{C} & -1.4365299 & -2.7325594 & -0.4940777 \\ \mathrm{C} & -0.9179206 & -4.0171443 & -0.7501064 \\ \mathrm{C} & -2.5579655 & -2.6486580 & 0.3585470 \\ \mathrm{C} & -1.4802001 & -5.1626633 & -0.1756451 \\ \mathrm{H} & -0.0566145 & -4.1342831 & -1.4097247 \\ \mathrm{C} & -3.1184116 & -3.7877128 & 0.9445248 \\ \mathrm{H} & -3.0034461 & -1.6746429 & 0.5717430 \\ -2.5801172 & -5.0508389 & 0.6787697 \\ \mathrm{H} & -1.0575635 & -6.1445707 & -0.3987694 \\ \mathrm{H} & -3.0217286 & -5.9426754 & 1.1276196\end{array}$

87

$\begin{array}{lrcr}2,6-\mathrm{Trip}_{2} \mathrm{C}_{6} \mathrm{H}_{3} \mathrm{TeI}, & \text { MARI-BP86/TZVP, Energy }= & -1421.455568459 \\ \mathrm{C} & -0.3631493 & 3.1868466 & -1.6479677 \\ \mathrm{C} & -0.1836779 & 1.7997219 & -1.5912433 \\ \mathrm{C} & -0.1052254 & 1.1560440 & -0.3340770 \\ \mathrm{C} & -0.2117177 & 1.8882842 & 0.8651442 \\ \mathrm{C} & -0.3957609 & 3.2824227 & 0.7587325 \\ \mathrm{C} & -0.4686516 & 3.9318575 & -0.4722068 \\ \mathrm{H} & -0.4200566 & 3.6733169 & -2.6238152 \\ \mathrm{H} & -0.4804959 & 3.8556532 & 1.6840363 \\ \mathrm{H} & -0.6094759 & 5.0130351 & -0.5145239 \\ \mathrm{C} & -0.1519130 & 1.3128924 & 2.2501537 \\ \mathrm{C} & 1.0739076 & 1.3051448 & 2.9557990 \\ \mathrm{C} & -1.3420529 & 0.8828433 & 2.8876494 \\ \mathrm{C} & 1.0821369 & 0.8575064 & 4.2841678 \\ \mathrm{C} & -1.2779867 & 0.4477927 & 4.2170579 \\ \mathrm{C} & -0.0763761 & 0.4250593 & 4.9356416 \\ \mathrm{H} & 2.0266794 & 0.8422863 & 4.8347552\end{array}$




\begin{tabular}{|c|c|c|c|}
\hline $\mathrm{H}$ & -2.1963682 & 0.1120468 & 4.7041268 \\
\hline $\mathrm{C}$ & -0.0768387 & 0.9895262 & -2.8542639 \\
\hline C & 1.1898082 & 0.8045743 & -3.4763575 \\
\hline C & -1.2539473 & 0.4680559 & -3.4672671 \\
\hline C & 1.2480617 & 0.0947092 & -4.6821056 \\
\hline C & -1.1310042 & -0.2341115 & -4.6712946 \\
\hline C & 0.1067863 & -0.4316641 & -5.2972409 \\
\hline $\mathrm{H}$ & 2.2166499 & -0.0529819 & -5.1662217 \\
\hline $\mathrm{H}$ & -2.0334728 & -0.6335114 & -5.1389230 \\
\hline $\mathrm{C}$ & -2.6907099 & 0.9133271 & 2.1711098 \\
\hline $\mathrm{H}$ & -2.4899521 & 1.0635542 & 1.0994148 \\
\hline $\mathrm{C}$ & 2.3774220 & 1.7837214 & 2.3201020 \\
\hline $\mathrm{H}$ & 2.1821858 & 1.9436881 & 1.2492389 \\
\hline C & -0.0157570 & -0.0672761 & 6.3756355 \\
\hline $\mathrm{H}$ & 1.0235746 & 0.0790244 & 6.7164848 \\
\hline C & 2.4659798 & 1.3953556 & -2.8850627 \\
\hline $\mathrm{H}$ & 2.2379387 & 1.7016028 & -1.8531352 \\
\hline C & -2.6371309 & 0.7069207 & -2.8682941 \\
\hline $\mathrm{H}$ & -2.4919075 & 1.0443049 & -1.8310251 \\
\hline $\mathrm{C}$ & 0.2174719 & -1.1967786 & -6.6086500 \\
\hline $\mathrm{H}$ & 1.2852872 & -1.1991947 & -6.8859717 \\
\hline C & -3.4722340 & -0.4054377 & 2.3084269 \\
\hline $\mathrm{H}$ & -2.8669134 & -1.2637574 & 1.9861962 \\
\hline $\mathrm{H}$ & -3.7894506 & -0.5859567 & 3.3466595 \\
\hline $\mathrm{H}$ & -4.3823701 & -0.3729368 & 1.6908816 \\
\hline $\mathrm{C}$ & -3.5425700 & 2.1051963 & 2.6521070 \\
\hline $\mathrm{H}$ & -3.7695484 & 2.0159000 & 3.7256005 \\
\hline $\mathrm{H}$ & -3.0205697 & 3.0603696 & 2.4979422 \\
\hline $\mathrm{H}$ & -4.4978318 & 2.1458835 & 2.1064642 \\
\hline $\mathrm{C}$ & -3.5021632 & -0.5645527 & -2.8217937 \\
\hline $\mathrm{H}$ & -3.7686551 & -0.9181235 & -3.8293038 \\
\hline $\mathrm{H}$ & -2.9833218 & -1.3783139 & -2.2964633 \\
\hline $\mathrm{H}$ & -4.4430477 & -0.3599777 & -2.2899268 \\
\hline C & -3.3664333 & 1.8368274 & -3.6242556 \\
\hline $\mathrm{H}$ & -2.7862114 & 2.7702156 & -3.6124381 \\
\hline $\mathrm{H}$ & -3.5369481 & 1.5589782 & -4.6755874 \\
\hline $\mathrm{H}$ & -4.3452379 & 2.0386934 & -3.1635720 \\
\hline C & 2.8229530 & 3.1347235 & 2.9143355 \\
\hline $\mathrm{H}$ & 2.0475466 & 3.9041288 & 2.7897329 \\
\hline $\mathrm{H}$ & 3.0318773 & 3.0409198 & 3.9908521 \\
\hline $\mathrm{H}$ & 3.7407064 & 3.4917644 & 2.4222349 \\
\hline C & 3.5051303 & 0.7412272 & 2.4293291 \\
\hline $\mathrm{H}$ & 3.8020911 & 0.5731452 & 3.4754062 \\
\hline $\mathrm{H}$ & 3.1970712 & -0.2260857 & 2.0089668 \\
\hline $\mathrm{H}$ & 4.3975232 & 1.0870153 & 1.8857373 \\
\hline C & 2.8889279 & 2.6598362 & -3.6611978 \\
\hline $\mathrm{H}$ & 3.1277762 & 2.4166951 & -4.7079160 \\
\hline $\mathrm{H}$ & 2.0916420 & 3.4165038 & -3.6646521 \\
\hline $\mathrm{H}$ & 3.7835730 & 3.1095094 & -3.2047790 \\
\hline $\mathrm{C}$ & 3.6217454 & 0.3814262 & -2.8208381 \\
\hline $\mathrm{H}$ & 3.3211174 & -0.5273148 & -2.2812377 \\
\hline $\mathrm{H}$ & 3.9681171 & 0.0890604 & -3.8237142 \\
\hline $\mathrm{H}$ & 4.4812251 & 0.8225503 & -2.2948478 \\
\hline $\mathrm{C}$ & -0.5566479 & -0.5018415 & -7.7446916 \\
\hline $\mathrm{H}$ & -0.2246866 & 0.5376542 & -7.8775558 \\
\hline $\mathrm{H}$ & -0.4067821 & -1.0341676 & -8.6959627 \\
\hline $\mathrm{H}$ & -1.6376220 & -0.4859783 & -7.5388785 \\
\hline $\mathrm{C}$ & -0.2258630 & -2.6638600 & -6.4529226 \\
\hline $\mathrm{H}$ & 0.3454492 & -3.1721561 & -5.6633737 \\
\hline $\mathrm{H}$ & -1.2928977 & -2.7313097 & -6.1922476 \\
\hline $\mathrm{H}$ & -0.0762629 & -3.2146849 & -7.3936661 \\
\hline
\end{tabular}




$\begin{array}{lrrr}\mathrm{C} & -0.9290800 & 0.7475589 & 7.3093394 \\ \mathrm{H} & -0.8137782 & 0.4150154 & 8.3521899 \\ \mathrm{H} & -0.6922995 & 1.8201863 & 7.2630502 \\ \mathrm{H} & -1.9885427 & 0.6254927 & 7.0374592 \\ \mathrm{C} & -0.3271398 & -1.5730774 & 6.4723933 \\ \mathrm{H} & -1.3616490 & -1.7836067 & 6.1611927 \\ \mathrm{H} & 0.3416269 & -2.1587067 & 5.8255666 \\ \mathrm{H} & -0.2079423 & -1.9306752 & 7.5066417 \\ \mathrm{Te} & 0.1754809 & -0.9611905 & -0.6932359 \\ \mathrm{I} & 0.4504875 & -2.2964264 & 1.7337804\end{array}$

26

$2-\mathrm{Me}_{2} \mathrm{NCH}_{2} \mathrm{C}_{6} \mathrm{H}_{4} \mathrm{TeN}_{3}$ ，MARI-BP86/TZVP, Energy =

$-3.1431467$

C $\quad-2.7187046 \quad-0.5301522 \quad-1.8805272$

$\begin{array}{llll}C & -1.8347987 & -0.4639264 & -0.7962918\end{array}$

$\begin{array}{llll}\text { C } & -0.5536721 & 0.0851519 & -0.9837177\end{array}$

C $\quad-0.1614452 \quad 0.5478708 \quad-2.2436835$

$\begin{array}{llll}\mathrm{C} & -1.0489080 & 0.4602291 & -3.3209542\end{array}$

$\mathrm{H}-\begin{array}{llll}-3.0228539 & -0.1333163 & -3.9828640\end{array}$

$\mathrm{H} \quad-3.7168473 \quad-0.9511800 \quad-1.7340332$

$\mathrm{H} \quad \begin{array}{rrrr}0.8357562 & 0.9705342 & -2.3765101\end{array}$

$\begin{array}{llll}\mathrm{H} & -0.7376972 & 0.8217538 & -4.3027166\end{array}$

$\begin{array}{llll}\text { C } & -2.1917386 & -1.0256126 & 0.5578804\end{array}$

$\mathrm{H} \quad-1.7344946 \quad-2.0234070 \quad 0.6715266$

$\mathrm{H} \quad-3.2866984 \quad-1.1395347 \quad 0.6713907$

$\begin{array}{llll}\mathrm{N} & -1.6327564 & -0.1784756 & 1.6372398\end{array}$

C $\quad-2.3530658 \quad 1.1037609 \quad 1.7555016$

$\mathrm{H} \quad-3.3965044 \quad 0.9434018 \quad 2.0861986$

$\mathrm{H} \quad \begin{array}{llll}\mathrm{H} & -1.8385791 & 1.7439097 & 2.4828490\end{array}$

$\begin{array}{llll}\mathrm{H} & -2.3584677 & 1.6091329 & 0.7824309\end{array}$

$\begin{array}{llll}\text { C } & -1.5822411 & -0.8831795 & 2.9290446\end{array}$

$\mathrm{H} \quad-1.0976308 \quad-0.2408177 \quad 3.6761334$

$\mathrm{H} \quad-2.5953995 \quad-1.1397057 \quad 3.2894056$

$\mathrm{H} \quad-0.9928880 \quad-1.8027177 \quad 2.8215338$

$\begin{array}{llll}\text { Te } & 0.7180413 & 0.2224880 & 0.7328477\end{array}$

$\begin{array}{llll}\mathrm{N} & 2.4833210 & 0.5810064 & -0.5172804\end{array}$

$\begin{array}{llll}\mathrm{N} & 3.1505728 & -0.4044987 & -0.8215079\end{array}$

$\mathrm{N} \quad 3.8491875 \quad-1.2588512 \quad-1.1579749$ 